$63^{\text {ème }}$ Congrès de la SFCO, 03023 (2015)

DOI:10.1051/sfco/20156303023

(C) Owned by the authors, published by EDP Sciences, 2015

\title{
POSTER
}

\section{La disjonction chirurgicale maxillaire rapide}

\author{
Dibatista J-C*, Courtois B**, Berenguier $\mathrm{D}^{* * *}$, Aldegheri $\mathrm{A}^{* * * * *}$ \\ * Chirurgien oral clinique du golfe de Saint-Tropez \\ ** Chirurgien oral MCU Toulouse \\ *** Orthodontiste clinique du golfe de Saint-Tropez \\ **** Chirurgien maxillo-facial coaching surg maxillo-facial bone center Marseille
}

Les anomalies transversales de la face sont fréquentes, et d'apparitions souvent précoces. Elles sont dominées par une insuffisance de croissance transversale basale du maxillaire. C'est l'endomaxillie, ou endognathie.

Troubles de l'articulé dentaire, arcade maxillaire plus étroite que la mandibulaire, positionnement de la langue perturbé (position basse ne lui permettant plus de jouer son rôle de conformateur palatin), troubles ventilatoires, sourire limité, inesthétique(avec des corridors sombres) sont presents.

La croissance transversale du maxillaire précède l'ossification définitive de la suture médio-palatine (14-15 ans chez les filles, 15-16 ans chez les garçons). Les anomalies qui en découlent sont rarement isolées, souvent accompagnées d'une dysmorphose tridimensionnelle complète verticale et sagittale.

Avant 14 ans, le traitement orthopédique seul, permet d'ouvrir la suture médio-palatine. Cette technique est décrite pour la première fois par ANGEL en 1860.

Au-delà, le potentiel de synostose des sutures est élevé, contrariant la disjonction orthopédique. Cette synostose est renforcée par le développement biomécanique du maxillaire, qui crée des résistances supplémentaires au niveau des piliers ptérygo- maxillaires et maxillo-malaires. L'expansion orthopédique est alors impossible.

La disjonction chirurgicale est une technique de choix chez les patients adultes ou en fin de croissance, pour traiter l'endomaxillie, car elle permet de supprimer toute résistance osseuse au déplacement latéral de l'un ou des 2 maxillaires.

C'est une technique conforme à la distraction osseuse, qui impose une période de latence post-opératoire permettant la formation d'un cal osseux précoce qui sera distracté, et favorisera angiogénèse et ostéogénèse au sein des sites osseux sectionnés.

Dans les situations de dysmorphoses complexes, la disjonction palatine est suivie secondairement d'une intervention type Lefort I d'avancé et/ou d'impaction/ou de bascule afin de palier le problème vertical et sagittal associés.

Les auteurs rapportent ici le cas d'une jeune patiente de 19 ans dont le motif de consultation était des douleurs au niveau des ATM.

L'examen endobuccal révèle l'existence d'un articulé inversé unilatéral, avec insuffisance transversale et une béance antérieure. Les examens complémentaires, radiologiques et moulages, confirment la dysmorphose : classe I squelettique avec endognathie maxillaire, une bascule palatine antihoraire associée à une latérodéviation mandibulaire, une classe II dentaire droite et classe III dentaire à gauche ainsi qu' un trouble fonctionnel ventilatoire.

Le choix thérapeutique s'oriente vers une disjonction inter-maxillaire, suivie d'un Lefort 1 de bascule et d'avancée.

This is an Open Access article distributed under the terms of the Creative Commons Attribution License 4.0, which permits unrestricted use, distribution, and reproduction in any medium, provided the original work is properly cited. 
Chronologie

Pose du disjoncteur par l’orthodontiste, chirurgie sous anesthésie générale,

activation du disjoncteur au 8ème jour postopératoire jusqu'au 21ème jour. Le disjoncteur est gardé en bouche jusqu'au 5ème mois, le temps de consolidation du cal osseux.

Après dépose du dispositif, l'orthodontiste règle partiellement le trouble occlusal, avant le deuxième temps opératoire.

En fin de traitement, l'équilibre dento-squelettique est rétabli, les troubles ventilatoires et les douleurs au niveau des atm supprimés.

Le succès d'une telle chirurgie n'est garanti que par la collaboration orthodontiste- chirurgien-patient.

Nom et adresse du conférencier

Jean Christophe DIBATISTA

Service de chirurgie orale

Clinique chirurgicale du golfe de Saint-Tropez

Rond point Diego Brosset

83580 Gassin (France)

jcdibatista@gmail.com 